\title{
A NOTE ON ABSTRACT INTEGRATION
}

\author{
BY \\ RICHARD B. DARST
}

I. Introduction. The principal purpose of this note is to distinguish between the basic nature of $S$-type (Stieltjes) and $L$-type (Lebesgue) integration with respect to a finitely additive set function (measure). Interest in these types of integration was given impetus by the fundamental representation theorem of Hildebrandt, Fichtenholz, and Kantorovitch (cf. [2]), and, since that time, a considerable amount of work has been done with these integrals along the lines of developing a formal theory and as a powerful analytic tool in the study of linear spaces.

In the case of the formal theory, the usual practice has been to start with a set algebra (ring) instead of a sigma algebra (ring) and define both of the integrals in this basic setting. (This is natural since the "integrator" need not be completely additive and, at any rate, on a sigma algebra the two integrals are, for essentially bounded functions, identical.) This procedure has worked well for the $S$-integral; however, for the $L$-integral a great deal of difficulty arises. For example, in this primitive setting, the $L$-integral (in general) is neither an absolutely continuous, nor a linear, nor a homogeneous operation. These difficulties, and the fact that, for a formal theory, no one of the three usual ways that the class of measurable functions can be defined is any more desirable than the others [in general, each yields a class of measurable functions distinct from the others], reflect the artificiality inherent in the usual ways of defining the class of measurable functions.

In this paper, we use a fourth class of function (called continuous) and, by considering the relationships between these four classes, establish a theorem (Theorem 2.2) that, in effect, says the natural setting of the L-type integral is a sigma algebra. Also, we show that the class of continuous functions (which includes the various types of bounded measurable functions) can be characterized entirely in terms of the $S$-integral (Theorem 2.1), and this, together with the definition of continuity, implies that the natural setting of the $S$-type integral is a set algebra. We conclude the paper by deriving a necessary and sufficient condition, in the case of a set algebra, in order that each continuous function belong to at least one of the classes of measurable functions and show, by example, that a set algebra need not be a sigma algebra in order to satisfy this condition.

This paper has been submitted to and accepted for publication by the Proceedings of the American Mathematical Society. It has been transferred to these Transactions, with the consent of the author, for technical reasons. Received by the editors July 5, 1960 and, in revised form, September 12, 1960. 
II. Notation and terminology. Throughout this paper we shall use the notation and terminology adopted in [1]. Hence, $(X, S)$ will denote a set algebra (of a set $X$ ) and $H(X, S)$ will denote the set of bounded finitely additive set functions on $S$. There are many ways in which a maximal proper ideal, in $S$, can be characterized and, for the purpose of this paper, we will use two characterizations: (1) a proper ideal $J$ in $S$ is maximal if and only if $E \in S$ implies one, and only one, of $E$ and $E^{\prime}=X-E \in J$, and (2) a subset $J$ of $S$ is a maximal proper ideal if and only if there exists (uniquely) $g \in H(X, S)$ such that $g(E)=0$ if $E \in J$ and $g(E)=1$ if $E \notin J$. If $g$ has the properties of (2) we say that $g$ is a two-valued jump function. For the definitions of the $S$ - and $L$-type integrals see [2] or [3]. All functions considered in this paper are assumed to be real valued.

Definition 2.1. If $f$ is a function on $X$, then $f$ is said to be an $(X, S)$ continuous function if, for each $e>0$, there exists a partition $\left\{E_{i}\right\}_{i=1}^{n}$ of $X$ such that $E_{i} \in S$ and $O\left(f, E_{i}\right)<e$ for $i \leqq n$ where $O\left(f, E_{i}\right)=\operatorname{lub}_{x, y \in E_{i}}|f(x)-f(y)|$ (i.e., $O(f, E)$ denotes the oscillation of $f$ on $E)$. The collection of $(X, S)$ continuous functions is denoted by $C(X, S)$.

Theorem 2.1. Let $(X, S)$ be a set algebra and let $f$ be a function on $X$. Then $f$ is an $(X, S)$-continuous function if and only if $s \int_{X} f d g$ exists for each $g \in H(X, S)$.

Proof. We will show that if $f$ is not $(X, S)$-continuous then there exists a two-valued jump function $g$ on $S$ such that $s \int_{X} f d g$ does not exist. If, for $e>0$, we let $D(f, S, e)=\left[E \in S\right.$; if $\left\{E_{i}\right\}_{i=1}^{n}$ is a partition of $E$ in $S$ (i.e., $E_{i} \in S$ for $i \rightarrow n$ ), then $\left.\operatorname{lub}_{i \leqq n} O\left(f, E_{i}\right) \geqq e\right]$, then, $f \in C(X, S)$ if and only if $D(f, S, e)=\theta$ for $e>0$. Suppose there exists $e>0$ such that $D(f, S, e) \neq \theta$. Let $I$ denote the set of ideals in $S$ such that $J \in I$ and $E \in J$ imply $E^{\prime}=X-E \in D(f, S, e)$. There exists $J \in I$ which is maximal with respect to inclusion. Suppose there exists $E \in S$ such that each of $E$ and $E^{\prime} \notin J$. Then each of $K$ and $K_{1}$, the ideals generated by $J$ and $E$ and $J$ and $E^{\prime}$ respectively (i.e., $F \in K$ if and only if there exist $G \in J$ and $H \in S$ such that $F=G \cup(H \cap E)$ ), is a proper ideal in $S$ which contains $J$ as a proper subset. Therefore, each of $K$ and $K_{1} \notin I$ and there exist $F \in K$ and $F_{1} \in K_{1}$ such that each of $F^{\prime}$ and $F_{1}^{\prime} \notin D(f, S, e)$ which imply each of $E^{\prime} \cap F^{\prime}$ and $E \cap F_{1}^{\prime} \notin D(f, S, e)$; however, each of $E^{\prime} \cap F$ and $E \cap F_{1} \in J$. But, $X=E \cup E^{\prime}=\left(E \cap F_{1}\right) \cup\left(E \cap F_{1}^{\prime}\right) \cup\left(E^{\prime} \cap F\right) \cup\left(E^{\prime} \cap F^{\prime}\right)$ $=\left(E \cap F_{1}\right) \cup\left(E^{\prime} \cap F\right) \cup\left(E \cap F_{1}^{\prime}\right) \cup\left(E^{\prime} \cap F^{\prime}\right),\left(E \cap F_{1}\right) \cup\left(E^{\prime} \cap F\right) \in J$ and $\left(E \cap F_{1}^{\prime}\right) \cup\left(E^{\prime} \cap F^{\prime}\right) \notin D(f, S, e)$. This contradiction shows our supposition that $J$ is not a maximal proper ideal in $S$ is false. There exists $g \in H(X, S)$ such that $g(E)=0$ if $E \in J$ and $g(E)=1$ if $E \notin J ; s \int_{X} f d g$ does not exist.

REMARK. Perhaps it is of interest to note that, in a sense, Theorem 2.1 is an extension to the general case of the classical theorem which states that a function $f$ on the interval $[a, b]$ is continuous if and only if the Stieltjes integral $\int_{a}^{b} f d g$ exists for every function $g$ of bounded variation on $[a, b]$. Also, we note that, regarding $C(X, S)$ as a linear-normed-complete space, $C(X, S)$ 
is isomorphically isometric to the space of topologically continuous functions on $\beta(S)$, where $\beta(S)$ is the space of ultrafilters associated with $S$ (i.e., the Stone-Cech type compactification of $(X, S)$ ). Finally, we see that a function $f$ on $X$ is in $C(X, S)$ if and only if there exists a sequence $\left\{f_{i}\right\}$ of $(X, S)$ simple functions such that $\lim _{i}\left\|f-f_{i}\right\|=\lim _{i}\left(\operatorname{lub}_{x \in X}\left|f(x)-f_{i}(x)\right|\right)=0$.

Definition 2.2. If $(X, S)$ is a set algebra, then

(1) $M(X, S)=\left[f\right.$ on $\left.X ;-\infty \leqq a<b \leqq \infty \Rightarrow f^{-1}(a, b) \in S\right], f^{-1}(a, b)$ $=[x \in X ; a<f(x)<b]$,

(2) $L M(X, S)=\left[f\right.$ on $\left.X ;-\infty<a<b \leqq \infty \Rightarrow f^{-1}[a, b) \in S\right], f^{-1}[a, b)$ $=[x \in X ; a \leqq f(x)<b]$,

(3) $R M(X, S)=\left[f\right.$ on $\left.X,-\infty \leqq a<b<\infty \Rightarrow f^{-1}(a, b] \in S\right], f^{-1}(a, b]$ $=[x \in X ; a<f(x) \leqq b]$,

(4) $G(X)=\left[f\right.$ on $\left.X ;\|f\|=\operatorname{lub}_{x \in X}|f(x)|<\infty\right]$, and

(5) $m(X, S)=G(X) \cap M(X, S), \quad \operatorname{Lm}(X, S)=G(X) \cap L M(X, S)$, and $R m(X, S)=G(X) \cap R M(X, S)$.

The following two lemmas follow readily from Definition 2.2.

Lemma 2.1. Let $(X, S)$ be a set algebra. Then

(1) if $\left\{E_{i}\right\}_{i=1}^{n}$ is a finite collection of pairwise disjoint subsets of $X$ and $\cup_{i \leq n} E_{i} \in S$ then either $E_{i} \in S$ for $i \leqq n$ or there exist at least two indices $i$ and $j$ such that each of $E_{i}$ and $E_{j} \notin S$,

(2) each of $m(X, S), \operatorname{Lm}(X, S)$, and $\operatorname{Rm}(X, S)$ is a subset of $C(X, S)$,

(3) if $f \in M(X, S)$ and $P$ is a real number then $f^{-1}(P) \in S$,

(4) $M(X, S)=L M(X, S) \cap R M(X, S)$, and

(5) if $f$ is a function on $X$, then $f \in M(X, S), L M(X, S)$, or $R M(X, S)$ if and only if $f^{n} \in m(X, S), \operatorname{Lm}(X, S)$, or $\operatorname{Rm}(X, S)$ respectively for each positive integer $n$ where $f^{n}(x)=f(x)$ if $|f(x)| \leqq n$ and $f^{n}(x)=n \cdot f(x) \cdot|f(x)|^{-1}$ if $|f(x)|>n$.

Leмma 2.2. If $(X, S)$ is a sigma algebra, then

(1) $M(X, S)=L M(X, S)=R M(X, S)$, and

(2) $m(X, S)=C(X, S)$.

Proof of (2). If $f \in C(X, S)$, then there exists a sequence $\left\{f_{n}\right\}$ of $(X, S)$ simple functions such that $\left\|f-f_{n}\right\|<n^{-1} ; a<b$ implies $f^{-1}(a, b)$ $=\bigcup_{j \geq 1} \bigcap_{i \geq j} f_{i}^{-1}\left(a+j^{-1}, b-j^{-1}\right)$.

TheOREM 2.2. Let $(X, S)$ be a set algebra, $m=m(X, S), \operatorname{Lm}=\operatorname{Lm}(X, S)$, $R m=R m(X, S)$, and $C=C(X, S)$. Then $C=m=L m=R m$ if and only if $(X, S)$ is a sigma algebra. Moreover, if $(X, S)$ is not a sigma algebra $L m \neq R m, m \subset L m$, $m \subset R m(m \neq L m, m \neq R m)$, and each of $L m$ and $R m$ is a proper subset of $C$.

Proof. If $(X, S)$ is not a sigma algebra then there exists a sequence $\left\{E_{i}\right\}$ of pairwise disjoint elements of $S$ such that $U E_{i} \notin S$. Let $f_{L}(x)=2^{-i}$ if $x \in E_{i}$ and $f_{L}(x)=0$ if $x \in X-U E_{i}$ and let $f_{R}=-f_{L} ; f_{L} \in L m-R m$ and $f_{R} \in R m-L m$ $(f \in L M(X, S)$ if and only if $-f \in R M(X, S))$. 
Definition 2.3. Let $(X, S)$ be a set algebra. Then by

(1) $\left\{E_{i}\right\} \uparrow$ in $S$ we mean that $\left\{E_{i}\right\}$ is a nondecreasing (i.e., $E_{i} \subset E_{i+1}$ ) sequence of elements of $S$,

(2) $\left\{E_{i}\right\} \downarrow$ in $S$ we mean that $\left\{E_{i}\right\}$ is a nonincreasing sequence of elements of $S$, and

(3) the statement that $S$ has property $Q$ we mean that if $\left\{E_{i}\right\} \uparrow$ in $S$, $\left\{F_{i}\right\} \uparrow$ in $S$ and $\left(U E_{i}\right) \cap\left(U F_{i}\right)=\theta$ then at least one of $U E_{i}$ and $U F_{i} \in S$.

Lemma 2.3. Let $(X, S)$ be a set algebra and let $S$ have property $Q$. Then

(1) if $\left\{E_{i}\right\} \downarrow$ in $S,\left\{F_{i}\right\} \uparrow$ in $S$, and there exists a positive integer $j$ such that $E_{j} \cap\left(U F_{i}\right)=\theta$ then at least one of $\cap E_{i}$ and $U F_{i} \in S$, and

(2) if $\left\{E_{i}\right\} \downarrow$ in $S,\left\{F_{i}\right\} \downarrow$ in $S$, and there exists a positive integer $j$ such that $E_{j} \cap\left(\cap F_{i}\right)=\theta$ then at least one of $\cap E_{i}$ and $\cap F_{i} \in S$.

Proof. (1) Let $G_{i}=E_{j}-E_{i} ;\left\{G_{i}\right\} \uparrow$ in $S$, $\left(U G_{i}\right) \cap\left(U F_{i}\right)=\theta$, and $\cap E_{i}=$ $=E_{j}-U G_{i}\left(\cap E_{i} \in S\right.$ if and only if $\left.\cup G_{i} \in S\right)$.

(2) $\cap F_{i}=\left(\cap F_{i}\right) \cap E_{j}^{\prime}=\cap\left(F_{i} \cap E_{j}^{\prime}\right)$. Let $G_{i}=F_{i} \cap E_{j}^{\prime}$ and $H_{i}=E_{j}-E_{i}$; $\left\{G_{i}\right\} \downarrow$ in $S,\left\{H_{i}\right\} \uparrow$ in $S, \cap G_{i}=\cap F_{i}, \cup H_{i}=E_{j}-\cap E_{i}$, and $G_{1} \cap\left(U H_{i}\right)=\theta$.

Lemma 2.4. Let $(X, S)$ be a set algebra, $S$ have property $Q$, and $f \in C(X, S)$. Then

(1) if each of $(a, b)$ and $(c, d)$ is a segment and $(a, b) \cap(c, d)=\theta$, then at least one of $f^{-1}(a, b)$ and $f^{-1}(c, d) \in S$,

(2) if $c \notin[a, b]$, then at least one of $f^{-1}(c)$ and $f^{-1}(a, b) \in S$,

(3) if $c \neq P$, then at least one of $f^{-1}(c)$ and $f^{-1}(P) \in S$.

Proof. Since $f \in C(X, S)$, there exists a sequence $\left\{f_{i}\right\}$ of $(X, S)$-simple functions such that $\left\|f-f_{i}\right\|<(2(i+1))^{-2}$ which implies $f^{-1}\left(a+2^{-1}\left((i-1)^{-1}+i^{-1}\right)\right.$, $\left.b-2^{-1}\left((i-1)^{-1}+i^{-1}\right)\right) \subset f_{i}^{-1}\left(a+i^{-1}, b-i^{-1}\right) \subset f^{-1}\left(a+2^{-1}\left(i^{-1}+(i+1)^{-1}\right)\right.$, $\left.b-2^{-1}\left(i^{-1}+(i+1)^{-1}\right)\right)$; this implies $\left\{f_{i}^{-1}\left(a+i^{-1}, b-i^{-1}\right)\right\} \uparrow$ in $S$ and $\mathrm{U}_{f_{i}^{-1}}\left(a+i^{-1}, b-i^{-1}\right)=f^{-1}(a, b)$. The preceding, together with property $Q$, shows (1). To get (2) we note that $f^{-1}(c)=\cap f_{i}^{-1}\left(c-i^{-1}, c+i^{-1}\right)=\cap E_{n}$ where $E_{n}=\bigcap_{i \leqslant n} f_{i}^{-1}\left(c-i^{-1}, c+i^{-1}\right)$ and then apply Lemma $2.3-1$ to $\left\{E_{n}\right\} \downarrow$ in $S$ and $\left\{f_{i}^{-1}\left(a+i^{-1}, b-i^{-1}\right)\right\} \uparrow$ in $S$. We get (3) in a similar fashion from Lemma 2.3-2.

Definition 2.4. If $(X, S)$ is a set algebra, $f$ is a real valued function on $X$, and $P$ is a real number, then $P$ is said to have property $U$ with respect to $f$ if at least one of the following conditions is satisfied: (1) $f^{-1}(P) \notin S$, (2) $e>0$ implies there exists $(a, b)$ such that $|P-a|<e,|P-b|<e$, and $f^{-1}(a, b) \notin S$.

Lemma 2.5. Let $(X, S)$ be a set algebra, $S$ have property $Q, f$ be a real valued function on $X, P$ be a real number such that $P$ has property $U$ with respect to $f$, and each of $a, b$, and $c$ be a real number distinct from $P$ such that $a<b$. Then each of $f^{-1}(c), f^{-1}(a, b), f^{-1}[a, b)$, and $f^{-1}(a, b] \in S$.

Proof. Parts (2) and (3) of Lemma 2.4 imply $f^{-1}(c) \in S$. If $P \notin(a, b)$, 
then $P \notin[a, b]$ and parts (1) and (2) of Lemma 2.4 imply $f^{-1}(a, b) \in S$. If $P \in(a, b)$ then each of $f^{-1}(-\infty, a), f^{-1}(a), f^{-1}(b)$, and $f^{-1}(b, \infty) \in S$ and, hence, $f^{-1}(a, b) \in S$ (Lemma 2.1-1). Finally, $f^{-1}[a, b)=f^{-1}(a) \cup f^{-1}(a, b) \in S$ and $f^{-1}(a, b]=f^{-1}(a, b) \cup f^{-1}(b) \in S$.

Lemma 2.6. Let $(X, S)$ be a set algebra, $S$ have property $Q$, and $f \in C(X, S)$ $-\operatorname{Lm}(X, S)$. Then there exists uniquely a point $P$ such that $(1) f^{-1}(P) \notin S$ and (2) $-\infty \leqq a<P$ implies $f^{-1}(a, P) \notin S$.

Proof. There exists $[c, d)$ such that $f^{-1}[c, d) \notin S$. We want to find a point $P$ in $[c, d]$ which has property $U$ with respect to $f$. If $f^{-1}(c) \notin S$, let $P=c$; suppose $f^{-1}(c) \in S$; then $f^{-1}(c, d) \notin S$, if $f^{-1}\left(2^{-1}[c+d]\right) \notin S$, let $2^{-1}[c+d]=P$; otherwise, exactly one of $f^{-1}\left(c, 2^{-1}[c+d]\right)$ and $f^{-1}\left(2^{-1}[c+d], d\right) \notin S$ (Lemma 2.4-1), denote that one by $\left(c_{1}, d_{1}\right)$ and repeat the preceding inductively. If there exists a positive integer $i$ such that $f^{-1}\left(2^{-1}\left[c_{i}+d_{i}\right]\right) \notin S$, fine; otherwise, let $P=\cap\left[c_{i}, d_{i}\right]$. It is impossible that $P \in(c, d)$ since $P \in(c, d)$ would imply $f^{-1}[c, d) \in S$ (Lemma 2.5). If $P=d$, then $f^{-1}(c) \in S$ (Lemma 2.5) which implies $f^{-1}(c, P) \notin S$ which, in turn, implies $f^{-1}(P, \infty) \in S$ (Lemma 2.4) and thus $f^{-1}(P)=\left(X-\left[f^{-1}(-\infty, c) \cup f^{-1}(c) \cup f^{-1}(P, \infty)\right]\right)-f^{-1}(c, \quad P) \notin S \quad$ (Lemma 2.1-1). If $P=c$, let $e$ be a number less than $P$. Then $f^{-1}(e, d) \in S$ (Lemma 2.5) which implies $f^{-1}(e, P)=f^{-1}(e, d)-f^{-1}[P, d) \notin S(P=c$, Lemma 2.1-1) which, in turn, implies $f^{-1}(P, d) \in S$ (Lemma 2.4-1) and thus $f^{-1}(P)=f^{-1}[P, d$ ) $-f^{-1}(P, d) \notin S$. The lemma now follows from Lemma 2.4 and Lemma 2.5.

REMARK. Since $f \in R m(X, S)$ if and only if $-f \in \operatorname{Lm}(X, S)$ we have a dual result for $f \in C(X, S)-R m(X, S)$ (i.e., there exists uniquely a point $P$ such that $f^{-1}(P) \notin S$ and $b>P$ implies $\left.f^{-1}(P, b) \notin S\right)$.

Theorem 2.3. Let $(X, S)$ be a set algebra. Then $C(X, S)=\operatorname{Lm}(X, S)$ $\cup R m(X, S)$ if and only if $S$ has property $Q$.

Proof. If $S$ has property $Q$ and $f \in C(X, S)-\operatorname{Lm}(X, S)$ then there exists uniquely a point $P$ satisfying the conditions of Lemma 2.6. Suppose $f \notin R m(X, S)$. Then there exists uniquely a point $P^{\prime}$ satisfying the conditions of the remark following Lemma 2.6; moreover, $P^{\prime}=P$ (Lemma 2.4-3). This contradicts the supposition that $f \notin R m(X, S)$ (Lemma 2.6 and the remark following Lemma 2.6, Lemma 2.4-1). If $S$ does not have property $Q$ then there exist sequences $\left\{E_{i}\right\} \uparrow$ in $S$ and $\left\{F_{i}\right\} \uparrow$ in $S$ such that $\left(U E_{i}\right) \cap\left(U F_{i}\right)=\theta$ and each of $U E_{i}$ and $U F_{i} \in S$. Let $f(x)=1$ if $x \in E_{1}, f(x)=2^{-i}$ if $i>1$ and $x \in E_{i}$ $-U_{j<i} E_{j}, f(x)=-1$ if $x \in F_{1}, f(x)=-\left(2^{-i}\right)$ if $i>1$ and $x \in F_{i}-U_{j<i} F_{j}$, and $f(x)=0$ if $x \in X-\cup\left(E_{i} \cup F_{i}\right) ; f \in C(X, S)-[\operatorname{Lm}(X, S) \cup R m(X, S)]$.

We conclude with an example to show that the property of being a sigma algebra (while sufficient) is not necessary in order that $S$ have property $Q$. Let $I$ be the set of positive integers, let $E \in T$ if and only if $E \subset I$, let $J$ be a maximal proper ideal in $T$ such that if $E$ is a finite subset of $I$ then $E \in J$, let $X=I+[0]$, and let $E \in S$ if and only if one of $E$ and $X-E \in J$ (i.e., we 
add 0 to the elements of $T-J) .(X, S)$ is a set algebra which is not a sigma algebra and such that $S$ does have property $Q$.

\section{BiBLIOGRAPHY}

1. R. B. Darst, $A$ decomposition of finitely additive measures, J. Reine Angew. Math., to appear.

2. T. H. Hildebrandt, On bounded linear functional operations, Trans. Amer. Math. Soc. vol. 36 (1934) pp. 868-875.

3. A. E. Taylor, Introduction to functional analysis, New York, John Wiley, 1958.

Louisiana State University,

BATON ROUGE, LOUISIANA

MassachusetTS INSTITUTE OF TeChNology,

Cambridge massachusetts 